\title{
Evaluation of Eddy Current Response Due to the Applied Stress on a Steel Plate Using Phase Diagram
}

\author{
Sanjeema BAJRACHARYA ${ }^{\mathrm{a}, 1}$ and Eiichi SASAKI ${ }^{\mathrm{a}}$ \\ a Tokyo Institute of Technology, Tokyo, Japan
}

\begin{abstract}
Structural health monitoring of steel structures is crucial for inspection of corrosion and cracking in structural members, compromising their safety and serviceability. In the present study, the prospective of evaluation of change in stress state of structural member due to corrosion and cracking through eddy current based stress measurement is investigated. For this, three-dimensional numerical simulations are carried out in the FE software COMSOL Multiphysics 5.2a for a steel plate subjected to change in relative permeability, representative of change in stress state, whereby the eddy current indices are characterized, including the effects of additional influential parameters namely, lift-off, excitation frequency, and probe size. Phase Diagram is then proposed as a concise method to evaluate the variation of relative permeability and lift-off concurrently in a single graph for an excitation frequency and probe size. It further facilitates the selection of suitable excitation frequency and probe size to conduct the eddy current based stress measurement.
\end{abstract}

Keywords. eddy current, stress measurement, numerical simulation, phase diagram.

\section{Introduction}

Steel structures forming an integral part of the infrastructure suffer from corrosion due to long-term exposure to harsh environment and cracking due to fatigue as they near the end of their design life. Corrosion in the steel plate girders results in the reduction in their load-bearing capacity [1], leading to safety concerns of the overall structure, while fatigue cracking results in sudden brittle fracture [2]. Hence, inspection and monitoring of the steel structures for evaluation of the extent of effect of corrosion and detection of cracking, under structural health monitoring, have become crucial.

Various non-destructive testing methods such as ultrasonic testing, X-ray diffraction, and eddy current testing have been developed and employed for structural health monitoring of steel structures [3-6]. Among these, the eddy current testing has been selected in the present study because it is a non-contact method that does not require the use of coupling agent nor surface preparation such as clearing off the top rust layer, thus proving itself time and cost efficient, particularly for field inspection of large structures with damages such as corrosion and cracking that are widespread $[6,7]$.

${ }^{1}$ Corresponding author, Tokyo Institute of Technology, 2-12-1 Ookayama, Meguroku, Tokyo, Japan; E-mail: bajracharya.s.aa@m.titech.ac.jp 
Conventionally, the extent of corrosion is determined by the remaining thickness of a structural member [6], while the cracks are detected by the change in eddy current signal due to the distortion of eddy current around them [7]. Nevertheless, it is to be noted that both corrosion and cracking cause change in stress distribution in the structural members, which can be detected by eddy current testing based on the Villari effect [8]. Hence, a more viable approach for evaluation of corrosion and cracking through the stress change in a structural member, hereby termed as eddy current based stress measurement is proposed in the present study.

Eddy current testing has been applied in the determination of the internal stresses in the mechanical equipment in the industry $[4,5]$ and tension force in PT tendons, prestressed bars and cables, etc. [9-12]. However, its application has not been tested yet in the evaluation of stress change due to corrosion and cracking in structural members. Moreover, the eddy current probes used are designed to encircle the tendons, bars, and cables, whereas, surface probes are required to move over the structural members for determination of corrosion and cracking. Hence, a methodology to conduct eddy current based stress measurement using a suitable eddy current probe needs to be devised.

In reference to the eddy current influential parameters, lift-off has been maintained at a constant minimum $[5,11]$ to ensure sufficient sensitivity to the change in stress due to load in the PT tendons and cables. But it is difficult to achieve in field inspections such as a corroded steel plate girder with a rust layer. Only [10] and [11] have considered the effect of frequency in view of the stress measurement, while it has been found in [10] that miniaturization of probe offers better sensitivity to stress. However, it might not hold true when factoring in the larger lift-offs due to rust layer in a corroded structural member. Hence, the effect of these influential parameters - lift-off, excitation frequency, and probe size should be investigated for the eddy current based stress measurement.

Hence, the prospective of evaluation of corrosion and cracking through eddy current based stress measurement is investigated in the present study. For this, three-dimensional numerical simulations are carried out in the $\mathrm{AC} / \mathrm{DC}$ module of the general purpose, $\mathrm{FE}$ software COMSOL Multiphysics 5.2a, where the change in relative permeability of a steel plate, representative of stress, is evaluated by using an eddy current probe. Furthermore, a new, concise method of representing the simultaneous variation of relative permeability and lift-off, corresponding to a particular frequency and probe size, called Phase Diagram is proposed for easier evaluation of the effect of change in these influential parameters on eddy current indices, thus obtaining the criteria for selection of excitation frequency and probe size for the eddy current based stress measurement.

\section{Stress Measurement by Eddy Current Testing}

Eddy current testing is based on the principle of Faraday's law of electromagnetic induction. When an alternating current is passed through a coil, it generates primary magnetic field, which when brought near a conducting material induces eddy currents. The change in a material property such as magnetic permeability in the test material affects the eddy current flow, which is then reflected in the eddy current signal. When the stress is applied to a magnetostrictive material such as steel, domain reorientation occurs, causing change in its magnetic permeability, by the phenomenon known as Villari or Inverse magnetostrictive effect, thus enabling eddy current to detect the change in relative permeability due to stress. This can also be illustrated by one of the equations for linear magnetostriction [9] as shown in Eq. (1). 


$$
B=d^{*} \psi+\mu^{\psi} H
$$

Where, $\psi$ is the stress, $\mathrm{B}$ and $\mathrm{H}$ are the magnetic flux density and applied magnetic field, respectively, $\mu^{\psi}$ is the magnetic permeability at constant stress, and $\mathrm{d}^{*}$ is a piezomagnetic cross coupling coefficient. Eq. (1) shows that change in stress causes change in the magnetic flux penetrating the test material, which in turn affects the eddy current. This has also been verified experimentally by Jiles et al. (2002) [13] from the changes in hysteresis loop on application of stress. For positive magnetostrictive materials like steel, magnetic permeability increases with the increase in tensile stress.

In this study, numerical simulations are first carried out using a steel plate subjected to variation of relative permeability, representative of applied stress, as well as the other influential factors namely, lift-off, excitation frequency, and probe size. Then, the eddy current indices - real and imaginary voltages, and phase detected by a reflection-type eddy current probe are used to characterize the eddy current response. From the results of this parametric study, a new, concise method called Phase diagram is proposed to observe the simultaneous variation of relative permeability and lift-off at an excitation frequency for a probe size, which proffers the criteria for the selection of suitable excitation frequencies and probe size for the eddy current based stress measurement.

\section{Finite Element Modeling}

The three-dimensional numerical simulations are carried out in the frequency domain of the AC/DC module of FE software, COMSOL Multiphysics 5.2a, where the relative permeability of the steel plate is increased in X-direction to represent the increase in applied tensile stress. The reflection probe, comprising of an outer excitation coil and an inner detecting coil, selected in the present study for its high gain, is used to characterize the eddy current indices - real and imaginary voltages, and phase. It is modeled by using two concentric, homogenized, multi-turn cylindrical coils as shown in Figure 1 (a).

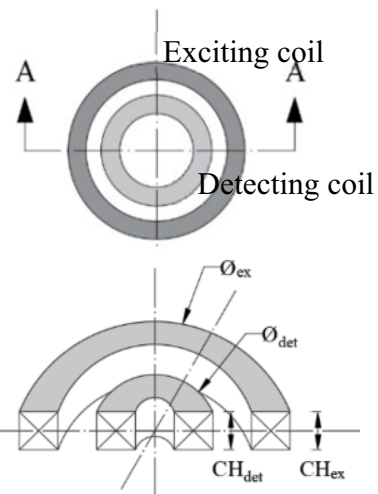

(a)

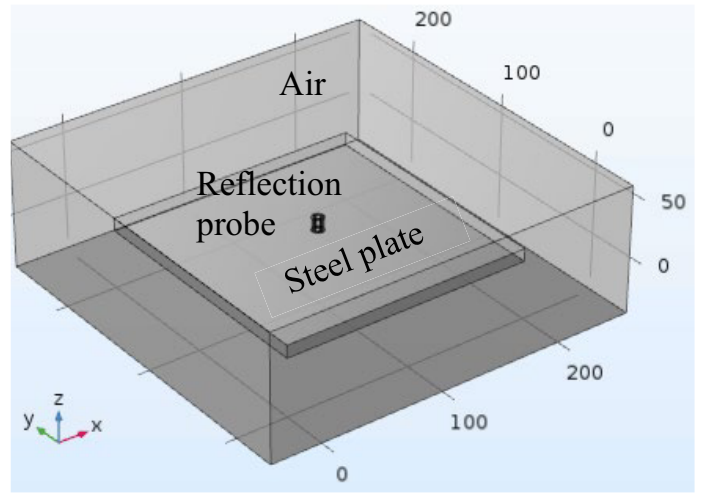

(b)

Figure 1. (a) Top view and cross-section of reflection probe, and (b) Finite element model

Here, $\phi$ is the coil diameter, $\mathrm{CH}$ is the coil height, and the subscripts ex and det denote exciting and detecting coils, respectively. The size of the computational domain 
(air) is $300 * 300 * 100 \mathrm{~mm}$ (Figure 1(b)) and the steel plate, made up of carbon steel, is $200 \mathrm{~mm} * 200 \mathrm{~mm}^{*} 10 \mathrm{~mm}$. The boundary condition is imposed such that the tangential component of the magnetic vector potential is zero. And, the material properties assigned to the steel plate, coils, and air are listed in Table 1.

Table 1. Material properties

\begin{tabular}{lrrr}
\hline \multicolumn{1}{c}{ Properties $\backslash$ Domain } & Air & Coils & \multicolumn{1}{c}{ Steel plate } \\
\hline Relative permeability along X-direction $\left(\mu_{\mathrm{rX}}\right)$ & 1 & 1 & $100-200$ \\
Electrical conductivity $(\sigma)$ & 0.1 & $5.998 * 10^{7}$ & $4.032^{*} 10^{6}$ \\
\hline
\end{tabular}

The parametric study is then conducted by varying the relative permeability of the steel plate along X-direction $\left(\mu_{\mathrm{rX}}\right)$ from 100 to 200 , lift-offs from 0 to $3 \mathrm{~mm}$, and excitation frequencies of $0.5,1$, and $5 \mathrm{kHz}$ for two probes $2 \mathrm{D} 1 \mathrm{CH}$ and $10 \mathrm{D} 10 \mathrm{CH}$ with exciting and detecting coils of diameters $2 \mathrm{~mm}$ and $1 \mathrm{~mm}$, and $10 \mathrm{~mm}$ and $6 \mathrm{~mm}$, respectively and coil heights of $1 \mathrm{~mm}$ and $10 \mathrm{~mm}$, respectively.

\section{Numerical Results and Discussion}

\subsection{Phase Diagram}

Instead of conventional practice of using individual eddy current indices to characterize the effect of change in influential parameters, a new, concise method, termed as Phase Diagram, is proposed to depict the variation of eddy current indices due to the concurrent changes in relative permeability and lift-off, at an excitation frequency for a probe size, in a single graph. It is constructed by plotting the real and imaginary voltages detected by the reflection probe along X-and Y-directions, respectively, as shown in Figure 2 for probe $2 \mathrm{D} 1 \mathrm{CH}$ at an excitation frequency of $1 \mathrm{kHz}$. In doing so, a clearer and succinct view of the change in more than one parameter at a time is obtained, therefore, condensing a number of graphs into one to extract the same amount of information as will be detailed in the following section. Thus, Phase diagram is used to evaluate the effect of the eddy current influential parameters henceforth in the present study.

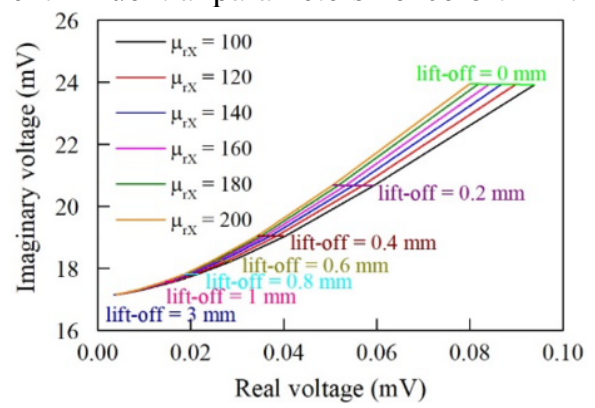

Figure 2. Phase diagram for probe $2 \mathrm{D} 1 \mathrm{CH}$ at excitation frequency of $1 \mathrm{kHz}$.

\subsection{Effect of Relative Permeability and Lift-off}

It can be seen from Figure 2 that real voltage decreases and phase increases with the increase in relative permeability in X-direction of the steel plate, while imaginary voltage 
is found to be less sensitive to change in relative permeability than the real voltage. Therefore, real voltage or phase could be an appropriate indicator for calibration in the future quantitative analysis. On the other hand, the well-known trend of eddy current that the detected voltages decrease as the lift-off increases is clearly observed; as the lift-off increases, the coupling between the probe and test material decreases, thus resulting in smaller value of detected voltage. In addition, the change due to relative permeability and lift-off can be easily distinguished from their distinct phase differences.

\subsection{Effect of Excitation Frequency and Probe Size}

Figure 3 shows the Phase diagrams for probe 10D10CH at excitation frequencies of 100 $\mathrm{Hz}, 1 \mathrm{kHz}$, and $5 \mathrm{kHz}$. It is clearly seen that at larger frequencies of 1 and $5 \mathrm{kHz}$, the trend of change in eddy current indices due to relative permeability becomes non-uniform for different lift-offs. Whereas, at a lower frequency of $100 \mathrm{~Hz}$, a uniform trend of change is obtained, which is invaluable for calibration of the eddy current indices for future quantitative analysis, also inferring that lower frequencies are more suited to conduct eddy current based stress measurement. However, comparing Figures 2 and 3(b), the larger probe $10 \mathrm{D} 10 \mathrm{CH}$ showed slightly inconsistent trend of change in eddy current indices at $1 \mathrm{kHz}$, while the smaller probe $2 \mathrm{D} 1 \mathrm{CH}$ gives a consistent trend of change at the same excitation frequency. Hence, the combined contribution of excitation frequency and probe size should be taken into account for selecting a suitable excitation frequency exhibiting consistent trend of change of relative permeability, for calibration purpose.

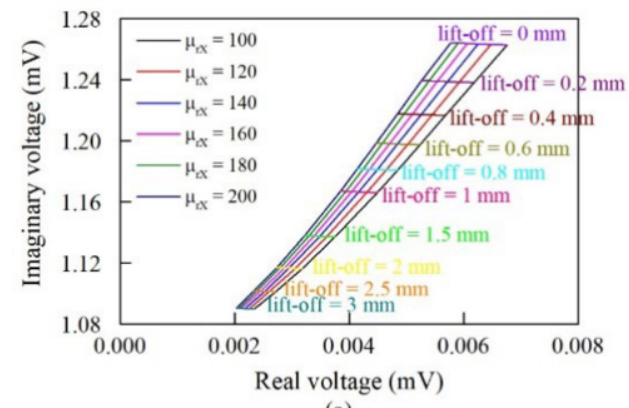

(a)

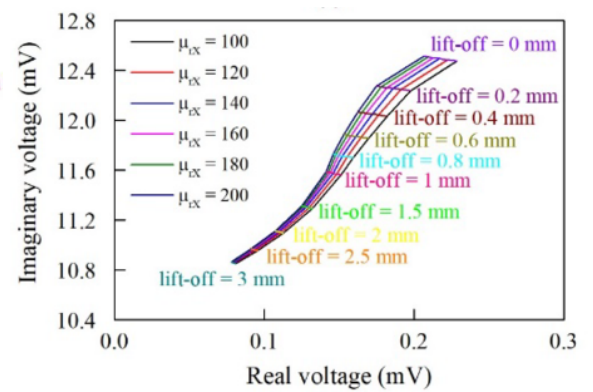

(b)

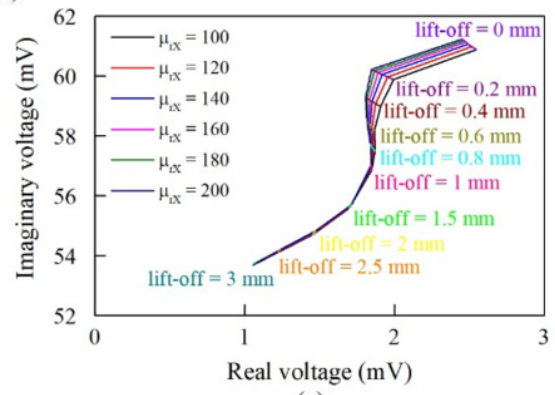

(c)

Figure 3. Phase diagram for probe $10 \mathrm{D} 10 \mathrm{CH}$ at excitation frequencies of (a) $100 \mathrm{~Hz}$,

(b) $1 \mathrm{kHz}$, and (c) $5 \mathrm{kHz}$.

Furthermore, the larger probe $10 \mathrm{D} 10 \mathrm{CH}$ showed sufficient sensitivity even at liftoffs as large as $3 \mathrm{~mm}$ (Figure 3), while the smaller probe showed highly diminished sensitivity at a $1 \mathrm{~mm}$ lift-off (Figure 2). Therefore, larger probes are deemed desirable to measure stress over a corroded surface with rust layer generating large lift-offs. 
Hence, the criteria for the selection of excitation frequency and probe size for the eddy current based stress measurement is obtained from the Phase diagram as the consistent trend of relative permeability requisite of calibration and sufficient sensitivity at different lift-offs.

\section{Conclusions}

In the present study, a comprehensive analysis of eddy current based stress measurement was carried out through three-dimensional numerical simulations in FE software COMSOL Multiphysics 5.2a. The effects of change in relative permeability of a steel plate due to the change in stress state in a steel plate and other influential factors namely, lift-off, excitation frequency, and probe size were characterized by using eddy current indices as detected by the reflection probe. Phase diagram was established as a viable, concise method of evaluating the concurrent change in the relative permeability and liftoff at an excitation frequency for a probe size. From the phase diagram, two important criteria for the selection of excitation frequency and probe size i.e., the uniform trend of change of eddy current indices, favoring lower excitation frequency, and sufficient sensitivity to larger lift-offs, respectively were obtained. The future works in this study would be evaluation of change in eddy current indices due to application of stress in multiple directions and experimental works offering comparison to the numerical results.

\section{Reference}

[1] N. Khurram, E. Sasaki, H. Katsuchi, and H. Yamada, Experimental and numerical evaluation of bearing capacity of steel plate girder affected by end panel corrosion, International Journal of Steel Structures 14 (2014), 659-676.

[2] T.L. Anderson, Fracture Mechanics: Fundamentals and Applications, CRC Press, Boca Raton, Florida, 2017.

[3] J. W. Wilson, G. Y. Tian, and S. Barrans, Residual magnetic field sensing for stress measurement, Sensors and Actuators A: Physical, 135 (2007), 381-387.

[4] J. Li, W. Zhang, W. Zeng, G. Chen, Z. Qiu, X. Cao, and X. Gao, Estimation of stress distribution in ferromagnetic tensile specimens using low cost eddy current stress measurement system and BP neural network, PloS ONE, 12 (2017), e0188197.

[5] A. Dahia, E. Berthelot, Y. Le Bihan, and L. Daniel, A model-based method for the characterisation of stress in magnetic materials using eddy current non-destructive evaluation, Journal of Physics D: Applied Physics, 48 (2015), 195002.

[6] A. Akutsu, E. Sasaki, Y. Ebisawa, and H. Tamura, Analysis of corrosion damage condition of steel members using low frequency eddy current testing, Journal of Japan Society of Civil Engineers, Ser. A1 (Structural Engineering \& Earthquake Engineering (SE/EE)), 73 (2017), 387-398.

[7] J. García-Martín, J. Gómez-Gil, and E. Vázquez-Sánchez, Non-destructive techniques based on eddy current testing, Sensors, 11 (2011), 2525-2565.

[8] B. D. Cullity and C. D. Graham, Introduction to magnetic materials, Wiley, Hoboken, New Jersey, 2009.

[9] J. M. Kim, J. Lee, and H. Sohn, Automatic measurement and warning of tension force reduction in a PT tendon using eddy current sensing, NDT\&E International, 87 (2017), 93-99.

[10] J.-G. Liu, and W.-J. Becker, Force and stress measurements with eddy-current sensors, Proceedings II of the SENSOR (2001), 23-28.

[11] W. Ricken, J.-G Liu, and W.-J Becker, GMR and eddy current sensor in use of stress measurement, Sensors and Actuators A: Physical, 91 (2001), 42-45.

[12] W. Ricken, H.-C. Schoenekess, and W.-J Becker, Improved multi-sensor for force measurement of prestressed steel cables by means of the eddy current technique, Sensors and Actuators A: Physical, 129 (2006), 80-85.

[13] D.C. Jiles, S.J., Lee, and C.C.H. Lo, The effects of stress on magnetic properties and the use of magnetic measurements for evaluation of materials, Conference on Resurgence of Metallic Materials (2002). 